\title{
Cadeira de Rodas e Estigma: um estudo preliminar da percepção visual de não- usuários
}

\section{Wheelchair end stigma: a preliminary study of visual perception of non-user}

Melissa Marin Vasquez ${ }^{1}$

Jamille Noretza de Lima Lanutti ${ }^{2}$

Fabiane Rodrigues Fernandes ${ }^{3}$

Fausto Orsi Mêdola ${ }^{4}$

Luis Carlos Paschoarelli ${ }^{5}$ 


\section{Resumo}

O Design Ergonômico analisa as relações entre as funções práticas, estéticas, simbólicas e emocionais, visando atender as necessidades e expectativas dos diferentes usuários. Este estudo teve como objetivo verificar a compreensão do estigma negativo de TAs, levando em consideração a influência da emoção do seu usuário por meio da aplicação (emprego) de um eyetracking de sistema remoto. Os resultados apontam que os sujeitos do gênero feminino são mais sensíveis a perceberem o estigma relacionado a tecnologia assistiva. Além disso destaca-se a método empregado como uma alternativa para obter dados quantitativos da percepção do valor simbólico negativo, ou seja o estigma social.

Palavras-chave: eyetracking, estigma, cadeira de rodas.

\section{Abstract}

The Ergonomic Design analyzes the relationship between the practical, aesthetic, symbolic and emotional functions in order to meet the needs and expectations of the various users. This study aimed to verify the understanding of the negative stigma of TAs, taking into account the influence of the emotion of their user through the application (employment) of an eyetracking remote system. The results show that the female subjects are more sensitive to realize the stigma related to assitiva technology. In addition there is the method used as an alternative to obtain quantitative data from the perception of negative symbolic value, that is the social stigma.

Key-words: eyetracking, stigma, Wheelchair.

ISSN: 2316.7963

1 Mestranda, FAAC-UNESP BAURU, SP,Brasil melissamava@gmail.com 2 Doutoranda, UNESP BAURU, SP,Brasil jamille lanutti@hotmail.com 3 Doutoranda, UNESP BAURU, SP,Brasil fabyfernandes@gmail.com
4 Prof. Dr., UNESP BAURU, SP,Brasil fausto.medola@faac.unesp.br 5 Prof. Dr.,UNESP BAURU, SP,Brasil paschoarelli@faac.unesp.br 


\section{Introdução}

O Design Ergonômico (DE) como subárea do Design, se preocupa em garantir a segurança, conforto e usabilidade das interfaces de uso humano. Estuda as relações de uso de um produto ou sistema, considerando suas funções práticas, estéticas e simbólicas, e analisa essas relações visando atender as necessidades e expectativas dos diversos usuários.

Quando os produtos e sistemas são destinados às pessoas com deficiência, o DE complementa os métodos projetuais empregados no desenvolvimento de Tecnologias Assistivas (TA), proporcionando alternativas para que, não apenas as funções práticas sejam atendidas, mas também as de ordem estética e simbólica.

As TAs apresentam expressivo valor simbólico e emocional enquanto produto de uso. Além disso desempenham um importante papel social - não só funcional para o usuário, os cuidadores, ou mesmo os usuários indiretos. Assim, estudos que tenham como objetivo o desenvolvimento de métodos para compreensão da significação das TAs representam uma demanda investigativa, cujos resultados podem contribuir expressivamente para o DE de TAs.

\section{Fundamentação teórica}

Segundo a OMS (Organização Mundial da Saúde) a deficiência é parte da condição humana. Isto significa que todas as pessoas estão susceptíveis a ter uma invalidez temporal ou permanente, em algum momento da vida. Como exemplo, pode-se apontar as pessoas que chegam a terceira idade e que experimentaram, progressivamente, as dificuldades do funcionamento corporal e cognitivo.

Um estudo realizado pela Carga Mundial de Mobilidade (2010), afirma que existem 975 milhões $(19,4 \%)$ de pessoas com deficiência; e estima-se que existem, aproximadamente, 190 milhões $(3,8 \%)$ de pessoas que apresentam alguma deficiência grave - tetraplegia, cegueira, entre outros.

Toda deficiência é complexa, dinâmica e objeto de discrepâncias. Associado à isto, diversas barreiras físicas e sociais interferem na cotidianidade de uma pessoa com deficiência. Algumas barreiras como a participação restringida nas atividades educativas, laborais e sociais são geradas pela falta de serviços de apoio (OMS, 2011).

Através das TAs, as pessoas com deficiência podem ser incluídas com maior facilidade na sociedade. De acordo com a ISO 9999 (2007), TA é definida como um "objeto fabricado especialmente e disponibilizado no mercado para prever, compensar, controlar e mitigar ou neutralizar deficiências, limitações em atividade e restrições na participação". Ainda, de acordo com essa norma, as TAs são elementos importantes para a pessoa com deficiência, uma vez que permites quem interajam com a sociedade, proporcionando funcionalidade, conforto e outras características físicas. Não obstante, este tipo de objeto tem uma carga simbólica muito forte e que pode ser negativa, afetando a reintegração social do usuário de TA.

A cadeira de rodas é uma das TAs mais utilizadas pela população com deficiência e $10 \%$ da população mundial tem ao longo da vida alguma experiência com este 
equipamento. Embora a cadeira de rodas seja um elemento que ajuda a pessoa com deficiência melhorar sua mobilidade, pode também ser vista como um elemento de estigma negativo tanto por parte do usuário como também da sociedade.

Alguns estudos têm relatado problemas de abandono das TAs, por parte de seus usuários diretos (HERRERA, et al., 2012; RIEMER-REISS e WACKER, 2000; PHILLIPS e ZHAO, 1993; PARETTE e SCHERER, 2004; VERZA, et al. 2006) e isto pode estar relacionado ao estigma que dispositivos assistivos podem proporcionar. Segundo $\mathrm{Pa}-$ rette e Scherer (2004), todas as pessoas com deficiência têm experimentado algum grau de preconceito causado pelo estigma em suas vidas. Isto decorre ao fato deste indivíduo apresentar capacidades diferentes dos demais considerados "normais", o que resulta em sentimentos de isolamento e exclusão, que podem partir dele próprio ou (e especialmente) das pessoas à sua volta.

Estes autores têm expressado que este estigma pode estar associado ao uso de TAs, como a cadeira de rodas, por exemplo, que são utilizadas por pessoas com problemas físicos e/ou cognitivos. Portanto este sentimento de exclusão por parte da sociedade também esta relacionado a mensagem de vulnerabilidade que a cadeira de rodas transmite, gerando barreiras para seus usuários. Por outro lado, a cadeira de rodas é a TA de uso mais frequente para melhorar a mobilidade de uma pessoa condição primordial para que possa gozar dos direitos humanos e de uma vida digna. Para as pessoas com deficiência, uma cadeira de rodas adequada pode estabelecer o primeiro passo para a inclusão e participação na sociedade (OMS, et al. 2008).

Além disso, existem alguns avanços no design da cadeira de rodas que demostram uma preocupação em "oferecer maior qualidade nos produtos, buscando ergonomia, eficiência, praticidade, conforto, durabilidade e acessibilidade" (MEDOLA, 2010), apesar de ainda existirem aspectos estéticos e simbólicos que têm sido pouco explorados, estudados e, portanto, conhecidos.

\section{O produto assistivo relacionado ao estigma.}

O produto cujo valor simbólico seja associado a um estigma negativo é socialmente carregado de conflitos (ou fatores sociais) referentes aos usuários, aos objetos, ao ambiente e aos transeuntes (BISPO e BRANCO, 2008). Nota-se ainda, que tal produto está relacionado a maneira como o mesmo é percebido ou "interpretado" socialmente, e que se relaciona à Semântica do Objeto.

Outro importante ponto a considerar neste contexto é o fato de que os objetos desempenham um papel importante na construção de identidade de quem o usa, e isto pode ser construído através dos símbolos que tais objetos possuem, sendo que alguns podem representar status, enquanto outros podem representar um estigma negativo (BISPO e BRANCO, 2008). Esses autores afirmam ainda que, no caso das pessoas com deficiência, os atributos que geram o estigma estão associados ao uso do objeto que estereotipa o usuário. Um exemplo é o da cadeira de rodas, cujo símbolo gera um conjunto de ideias preconceituosas sobre o papel social da pessoa com deficiência.

Outro exemplo está nos produtos de proteção médica, sobre os quais Vaes, et al. (2012) realizaram estudo no qual discutiram que este tipo de objetos, e demais 
objetos médico-hospitalares, tem uma história de rechaço, ao estar filiados a significados indesejáveis.

Luborsky (1993) afirma que muitos usuários e seus familiares expressam descontentamento com relação às TAs e ao significado cultural que tais produtos carregam. Alguns reclamam sobre características funcionais e sobre sua estética. Não obstante, também existem reclamações sobre as implicações culturais e sociais atribuídas aos usuários, e que leva muitas vezes ao abandono da TA (PARETTE e SCHERER, 2004; LUBORSKY, 1993; COSTA et.al, 2010).

Segundo Vaes (2014), quando um usuário recebe uma cadeira de rodas esteticamente diferente - com cores fortes, estampas, entre outros elementos diferenciados - pode relacioná-la com uma emoção positiva, o que pode gerar outros significados, e que podem ser transmitidos à sociedade.

Merleau-Ponty (1999) afirma que para haver significação antes é preciso que se perceba o fenômeno, que o autor chama de experiência perceptiva, por isso a percepção sempre ocorrerá no campo do "se". A percepção do objeto está correlacionada tanto com a linguagem ou forma, como com a memória, conforme afirma Silveira (2012, p.6) quando diz que a percepção de um sujeito é "mediada pelo que ele vê; pelo que ele já viu; pelo que ele recorda (memória) e pelas alterações que ocorrem no interior e no exterior do sujeito; neste último caso, o ambiente". Merleau-Ponty (1999) finaliza concluindo que aquele que percebe o faz com a espessura histórica, resgata uma tradição perceptiva e confronta o presente.

Brookes (1998) explica que as TAs se transformam em sinais que enviam mensagens; e que são percebidas pelos transeuntes como uma característica pouco comum numa pessoa e, portanto, transmite a ideia de que deve-se ter um comportamento diferente em torno dessa pessoa.

Desmet (2000) desenvolveu uma pesquisa com enfoque no Design baseada na emoção relacionada a cadeiras de rodas difereciadas. $O$ autor destaca que este objeto não é um objeto agradável e por esse motivo é importante o desenvolvimento de pesquisas que permitam auxiliar aos designers no projeto de melhores TAs.

\section{Objetivo}

Este estudo tem como objetivo verificar a compreensão do estigma negativo de TAs, levando em consideração a influência da emoção do seu usuário por meio da aplicação (emprego) de um eyetracking de sistema remoto. Dessa forma, procura fortalecer a compreensão das funções simbólicas relacionadas à TA cadeira de rodas, ao gerar dados que, futuramente podem gerar requisitos de projeto do DE.

\section{Materiais e métodos}

\section{Participantes}

Por se tratar de um estudo preliminar, participaram do estudo 4 (quatro) indivíduos, divididos em dois grupos: 2 (dois) do gênero feminino (idade média 19 d.p. 0) e 2 (dois) do gênero masculino (idade média 24,5 d.p. 3,5). Todos os sujeitos foram 
recrutados no campus da UNESP de Bauru e participaram de forma voluntaria, assinando o termo de consentimento livre e esclarecido (Resolução 466/2012-CNS-MS e o "Código de Deontologia do Ergonomista Certificado - Norma ERG BR 1002 ABERGO"), informando os objetivos e procedimentos do estudo.

\section{Materiais e equipamentos}

Para o desenvolvimento do estudo utilizou-se eyetracking - sistema remoto de rastreamento ocular não-invasivo - da marca TheEyeTribe ${ }^{\circledR}$ (Figura 1), capaz de registrar o olhar por meio de infravermelho. O eyetracking foi conectado a um computador onde foram apresentadas 4 (quatro) imagens, cada uma individualmente.

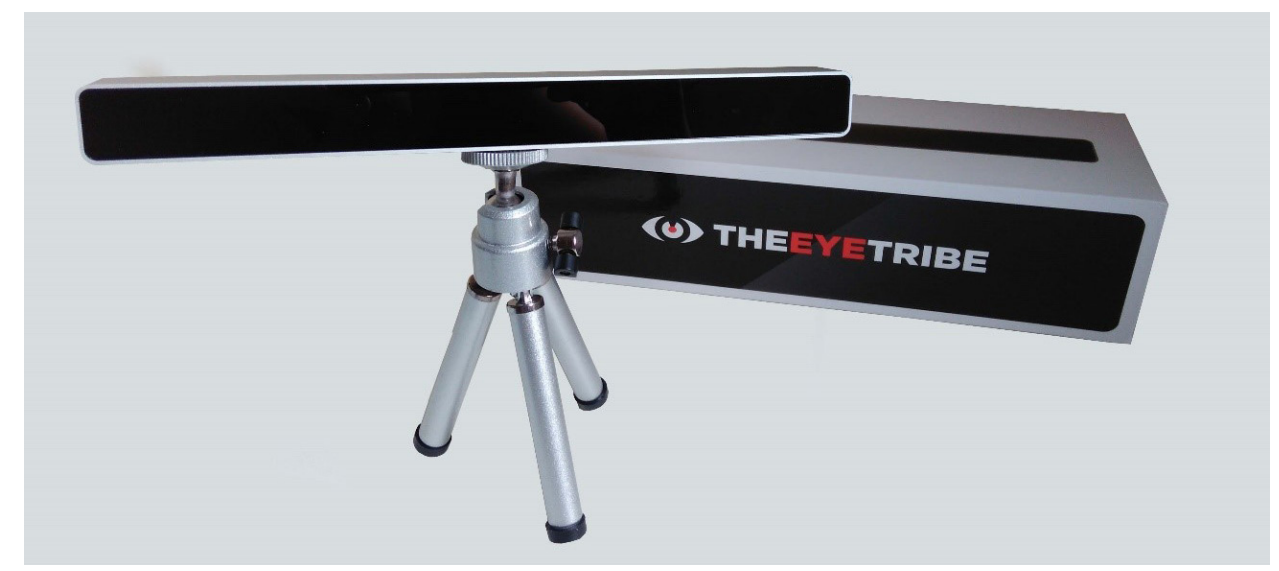

Figura 1- eyetracking TheEyeTribe®

As imagens apresentadas durante o estudo tratavam de dois diferentes casais, um com um dos sujeitos sentado em uma cadeira de rodas, e o outro com os dois sujeitos em pé. Duas imagens de cada casal com expressões emocionais opostas foram utilizadas: alegria e tristeza (Figura 2). Para produção destas imagens ainda foram utilizadas câmera Canon® e uma cadeira de rodas modelo Starlite (ORTOBRAS). 

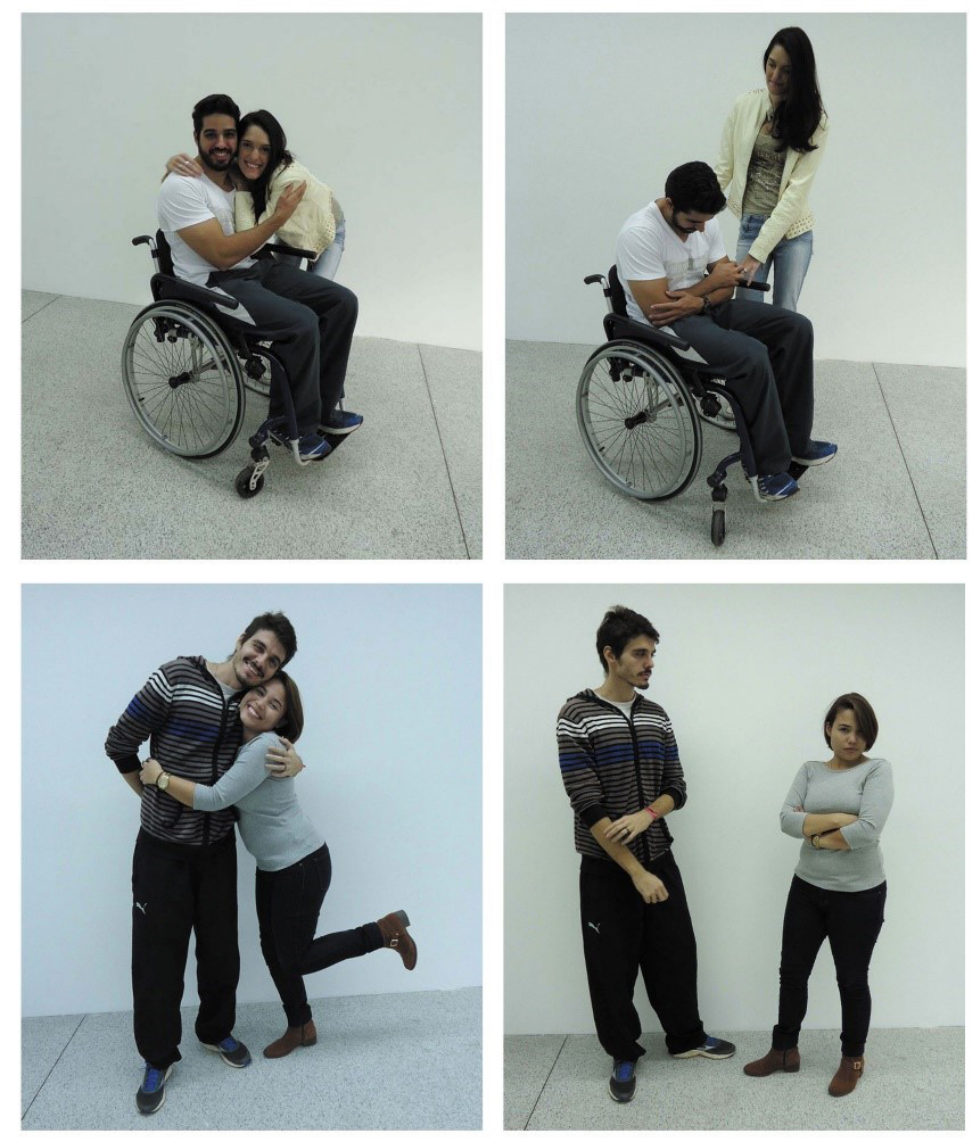

Figura 2- Imagens utilizadas no experimento.

\section{Procedimento}

O estudo foi realizado na Sala de Experimentos do Laboratório de Ergonomia e Interfaces da Unesp - Bauru, em um espaço neutro para não alterar a concentração e a percepção dos sujeitos.

Após o preenchimento do protocolo de identificação e do Termo de Consentimento Livre e Esclarecido, foi realizada a calibração do equipamento antes de iniciar o teste. Em seguida, os participantes recebiam uma mensagem para que mantivessem os olhos na tel(figura 3)a, e posteriormente, observavam cada uma das imagens, em um intervalo de 10 segundo cada (figura 4). Todos os procedimentos foram realizados com os participantes sentados em uma cadeira, em frente ao computador.

Observe as imagens a seguir. Se possivel não retire os olhos da tela.

Figura 3 - Mensagem inicial do estudo. 


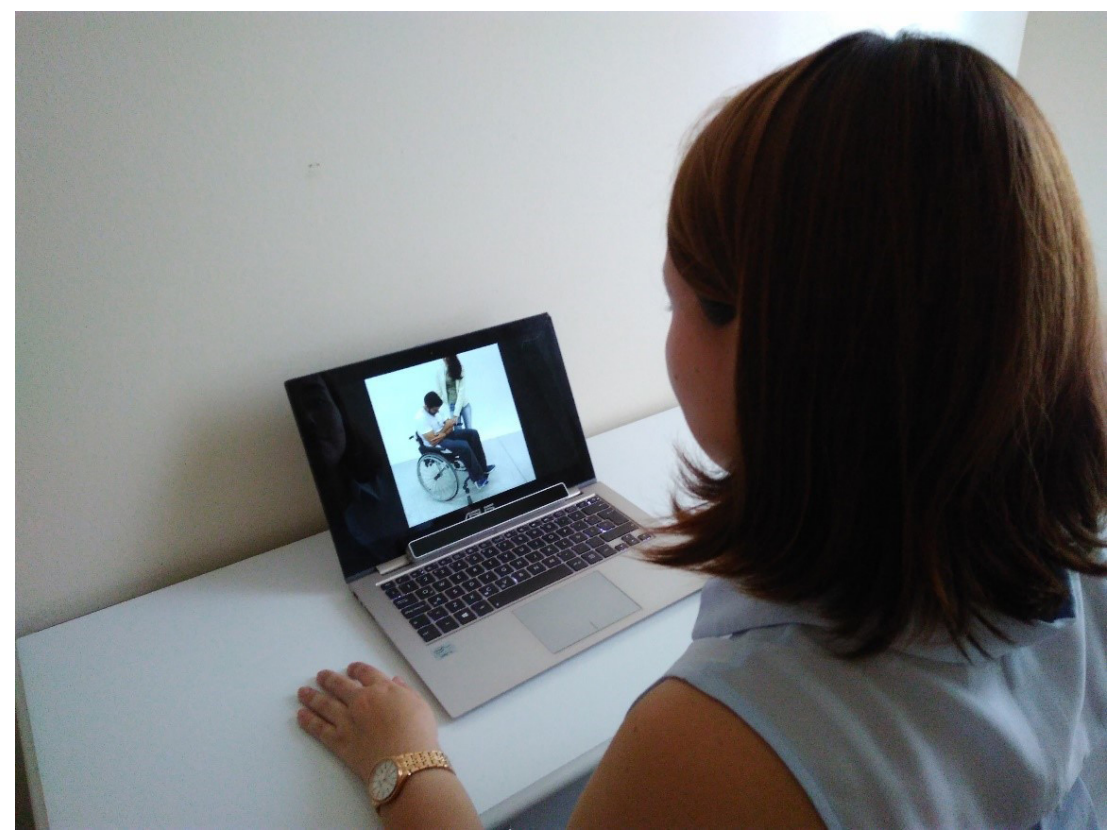

Figura 4- Sujeito observando as imagens durante a avaliação com eyetracking.

\section{Análise dos dados}

A análise dos dados foi realizada por meio do aplicativo de código aberto OGAMAC v. 5.0 desenvolvido pela Freie Universität Berlin, que permitiu a definição de duas áreas de interesse: a primeira tendo como foco a expressão que as pessoas apresentavam nas imagens utilizadas no estudo; e a segunda dando foco para as regiões da cadeira ou para as pernas, para as imagens com e sem a cadeira de rodas respectivamente (figura 5).
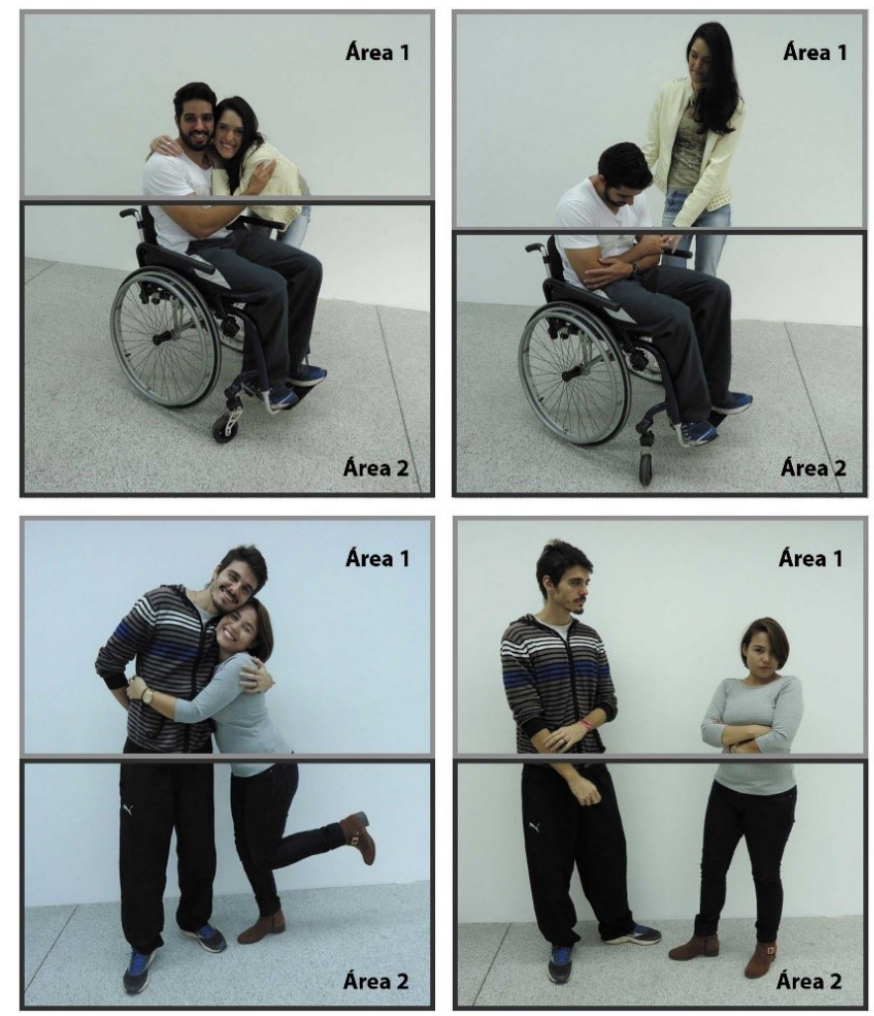

Figura 5 - Imagens utilizadas no experimento com áreas de interesse delimitadas. 
Assim, o referido software permitiu a comparação das diferentes áreas definidas por meio das seguintes variáveis: tempo de fixação, número de fixações e primeira fixação, definidos na tabela 1.

\begin{tabular}{|c|c|}
\hline $\begin{array}{c}\text { Tempo de } \\
\text { Fixação }\end{array}$ & Soma do tempo de fixação por Área pré-definida. \\
\hline $\begin{array}{c}\text { Número de } \\
\text { Fixações }\end{array}$ & Soma do número de fixações por Area pré-definida. \\
\hline $\begin{array}{c}\text { Primeira } \\
\text { Fixação }\end{array}$ & Ärea da imagem na qual ocorreu a primeira fixação. \\
\hline
\end{tabular}

Dessa forma, os dados coletados foram organizados em planilhas do Microsoft Excel ${ }^{\circ}$, tendo sido realizadas somas dos Tempos de fixação e do Número de fixação por gênero e de acordo com as áreas de interesse (A1 e A2) para cada imagem (conforme figura 5), a fim de compreender a percepção visual dos sujeitos. Além disso, o software utilizado permitiu conhecer qual a área onde ocorreu a primeira fixação do olhos.

\section{Resultados}

Após a análise dos dados pode-se observar que quanto a imagem do 'casal com usuário de cadeira de rodas', tanto triste quanto feliz, os sujeitos do gênero feminino fixaram mais vezes seu olhar na cadeira de rodas (Área 2) (tabela 2). No entanto, este comportamento é ainda mais ressaltado quando a emoção expressada pelo casal é a tristeza, ao que se observa que quando a emoção é negativa a cadeira de rodas chama mais atenção. 


\begin{tabular}{|c|c|c|c|c|c|c|c|c|}
\hline \multicolumn{9}{|c|}{ Análise da percepção de sujeitos considerando a fixação } \\
\hline \multirow[b]{2}{*}{ Gênero } & \multirow[b]{2}{*}{ Sujeito } & \multirow[b]{2}{*}{$\begin{array}{l}\text { Áreas } \\
\text { de } \\
\text { interesse }\end{array}$} & \multicolumn{2}{|c|}{$\begin{array}{c}\text { Casal Cadeirante } \\
\text { Triste (CCT) }\end{array}$} & \multicolumn{2}{|c|}{$\begin{array}{c}\text { Casal Cadeirante } \\
\text { Feliz (CCF) }\end{array}$} & \multirow{2}{*}{$\begin{array}{l}\text { Primeira } \\
\text { Fixação }\end{array}$} & \multirow{2}{*}{$\begin{array}{c}\text { Imagem } \\
\text { apresentada } \\
\text { primeiro }\end{array}$} \\
\hline & & & $\begin{array}{l}\text { Tempo } \\
\text { de } \\
\text { fixação }\end{array}$ & $\begin{array}{c}\text { Número } \\
\text { de } \\
\text { fixação }\end{array}$ & $\begin{array}{c}\text { Tempo } \\
\text { de } \\
\text { fixação }\end{array}$ & $\begin{array}{c}\text { Número } \\
\text { de } \\
\text { fixação }\end{array}$ & & \\
\hline \multirow{3}{*}{ F } & \multirow{2}{*}{ S1 } & A1 & 6169 & 16 & 5223 & 8 & A1 & $\mathrm{CCF}$ \\
\hline & & $\frac{A 2}{A 1}$ & $\frac{2261}{3389}$ & $\frac{21}{11}$ & 3330 & $\frac{12}{10}$ & \multirow[b]{2}{*}{ A1 } & \multirow[b]{2}{*}{ CCT } \\
\hline & S2 & A2 & 5104 & 16 & 240 & 1 & & \\
\hline \multirow{4}{*}{ M } & \multirow{2}{*}{ S3 } & A1 & 5652 & 10 & 5602 & 11 & \multirow[b]{2}{*}{ A1 } & \multirow[b]{2}{*}{ CCT } \\
\hline & & A2 & 3602 & 9 & 1565 & 7 & & \\
\hline & \multirow{2}{*}{ S4 } & A1 & 3360 & 11 & 6742 & 8 & \multirow{2}{*}{ A2 } & \multirow{2}{*}{$\mathrm{CCF}$} \\
\hline & & A2 & 5293 & 20 & 2403 & 7 & & \\
\hline & & & \multicolumn{2}{|c|}{ Casal Triste (CT) } & \multicolumn{2}{|c|}{ Casal Feliz (CF) } & \multirow[b]{2}{*}{$\begin{array}{l}\text { Primeira } \\
\text { Fixação }\end{array}$} & \multirow[b]{2}{*}{$\begin{array}{l}\text { Imagem } \\
\text { apresentada } \\
\text { primeiro }\end{array}$} \\
\hline Gênero & Sujeito & $\begin{array}{l}\text { Äreas de } \\
\text { interesse }\end{array}$ & $\begin{array}{l}\text { Tempo } \\
\text { de } \\
\text { fixação }\end{array}$ & $\begin{array}{c}\text { Número } \\
\text { de } \\
\text { fixação }\end{array}$ & $\begin{array}{l}\text { Tempo } \\
\text { de } \\
\text { fixação }\end{array}$ & $\begin{array}{c}\text { Número } \\
\text { de } \\
\text { fixação }\end{array}$ & & \\
\hline \multirow{4}{*}{$F$} & \multirow{2}{*}{ S1 } & A1 & 5723 & 9 & 6275 & 15 & \multirow{2}{*}{$\mathrm{A} 1$} & \multirow{2}{*}{ CT } \\
\hline & & A2 & 2929 & 5 & 2207 & 10 & & \\
\hline & \multirow{2}{*}{ S2 } & A1 & 5374 & 15 & 6562 & 15 & \multirow{2}{*}{ A1 } & \multirow{2}{*}{$\mathrm{CF}$} \\
\hline & & A2 & 3261 & 13 & 4603 & 6 & & \\
\hline \multirow{4}{*}{ M } & \multirow{2}{*}{ S3 } & A1 & 5139 & 7 & 7068 & 10 & 47 & $C F$ \\
\hline & & A2 & 3738 & 14 & 1997 & 8 & A2 & $\mathrm{CF}$ \\
\hline & & A1 & 2909 & 10 & 0 & 0 & & \\
\hline & $\$ 4$ & A2 & 2511 & 9 & 8835 & 20 & A1 & CI \\
\hline
\end{tabular}

Tabela 2 - Resultados e Análise da percepção de sujeitos considerando a fixação.

No que diz respeito aos sujeitos do gênero masculino, ocorreu também fixação na área da cadeira (A2) por mais tempo por um dos sujeitos (S2), isto pode ter sido influenciado pelo fato deste sujeito ter observado primeiro a imagem do 'casal com cadeira de rodas' triste.

Ainda para o gênero masculino, no que se refere as imagens do 'casal cadeirante', um dos sujeitos (S4) também fixa por mais tempo e tem maior número de fixações na cadeira de rodas (A2) quando a emoção expressada é a tristeza.

Assim, de forma geral, no que diz respeito ao 'casal cadeirante', quando a emoção expressada foi a tristeza os sujeitos em sua maioria tenderam a fixar mais o olhar na cadeira de rodas (A2) e não no rosto (A1). Porém, observaram primeiro o rosto do casal e depois a cadeira de rodas.

Quanto à imagem do casal 'não cadeirante', tanto triste quanto feliz, os sujeitos do gênero feminino geralmente fixaram seu olhar mais vezes e por mais tempo sobre as áreas de expressão (A1).

Quanto à percepção dos sujeitos de gênero masculino, apenas um sujeito (S3) fixou os olhos mais vezes na região da cintura para baixo (A2) quando a emoção expressa era a tristeza. Enquanto outro sujeito (S4) fixou o olhar por mais tempo e teve mais pontos de fixação da cintura para baixo (A2) quando a emoção expressada na 
imagem era a felicidade.

Contudo, destaca-se novamente que as imagens foram apresentadas aleatoriamente, e ainda que isso não tenha influenciado a percepção dos sujeitos de gênero feminino, pode ter sido um fator relevante para os sujeitos de gênero masculino. Assim, destaca-se que o sujeito S3 visualizou primeiro a imagem do 'casal feliz', observando mais e por mais tempo a região do rosto (A1). Dessa forma quando a segunda imagem foi apresentada, este fixou mais pontos na área abaixo da cintura (A2), explorando toda a imagem, já que se tratava do mesmo casal.

O mesmo ocorreu com o sujeito S4 que tendo visualizado primeiro o casal triste visualizou mais pontos de fixação e por mais tempo a região do rosto (A1), dando assim mais atenção a região abaixo da cintura (A2) ao observar a segunda imagem.

Assim, de forma geral, no que diz respeito ao 'casal não cadeirante', a emoção expressa parece não ter influenciado nem os sujeitos do gênero feminino, nem os sujeitos do gênero masculino.

\section{Discussão e Considerações Finais}

Este é um estudo experimental e que tem como foco a compreensão do estigma relacionado a TA e, que também testou a aplicação de um método de análise do comportamento visual - eyetracking - utilizando um dos mais representativos dispositivos de TA: a cadeira de rodas

Vale ressaltar que a utilização de imagens para abordar a percepção sobre a cadeira de rodas e seu usuário tem sido empregado em diversos estudos (BERND et al., 2011; GETTIHNG, 2006; WANG et al, 2011; VASQUEZ, et al., 2015; LANUTTI et al., 2015).

O presente estudo encontrou que a cadeira de rodas exerce influência na percepção visual de sujeitos não usuários quando apresentados a imagens similares que diferem principalmente com relação à presença ou não deste dispositivo de TA. Ain$\mathrm{da}$, os resultados apontam que, de uma forma geral, a cadeira de rodas capta maior atenção em um contexto de tristeza, o que sugere uma correlação entre a TA e emoções negativas.

Os resultados sugerem que os sujeitos do gênero feminino são mais sensíveis a perceberem o estigma relacionado à tecnologia assistiva. Percepções mais negativas do gênero feminino relacionadas à cadeira de rodas foram reportadas em um estudo anterior (LANUTTI et al., 2015).

Quanto a ferramenta utilizada o software do equipamento TheEyeTribe ${ }^{\circledR}$ apresentou algumas dificuldades para salvar os dados coletados devido à dependência do mesmo com a conexão de internet. Além disso, os dados coletados por meio do software do eyetracking não foram passiveis de análise por não serem claros.

Destacando-se a importância do software OGAMAC v. 5.0 para a análise dos dados deste estudo. Este software se adapta a diferentes tipos de eyetracking, além de não precisar de uma conexão da internet para guardar e analisar dados, o que permite que o estudo seja feito em qualquer lugar.

Neste contexto, ressalta-se que o OGAMA@ é um software livre, o que permite o uso de dados sem estar sujeito à condições comerciais. Além, de possibilitar que ou- 
tras pessoas aprimorem o sistema, permitindo inclusive o desenvolvimento do mesmo para diferentes aplicações, o que certamente auxilia no amplo desenvolvimento de novas pesquisas.

Portanto, recomendam-se algumas possibilidades de aperfeiçoamento para o método apresentado. Assim sendo, recomenda-se a aplicação do método aqui proposto com maior número de sujeitos de ambos os gêneros e também cadeirantes ou não.

Além disso, observou-se que ter utilizado as mesmas pessoas expressando tristeza e alegria para as imagens de casais não cadeirantes pode ter influenciado nos resultados, principalmente no que diz respeito aos sujeitos do gênero masculino. Desse modo, recomenda-se a mudança das pessoas para cada imagem a ser apresentada. Contudo, vale ressaltar que este estudo acertou ao mostrar as imagens de forma randômica, pois a ordem com que as emoções são mostradas poderia representar um viés nos resultados caso isso não tivesse sido feito.

Futuros estudos também podem ser realizados demonstrando 'cadeirantes' e 'não cadeirantes' de diferentes gêneros isoladamente, ou ainda pessoas com diferentes faixas etárias, objetivando compreender que fatores relacionados aos sujeitos que utilizam a TA estão envolvidos na percepção de quem observa.

Este estudo permite ainda a reflexão acerca da aplicação de diferentes ferramentas que permitam a análise quantitativa de critérios subjetivos, tais como valor simbólico e emoção, e que estão relacionados a produtos do dia-a-dia, tenham estes estigma negativo ou positivo.

Por fim, ressalta-se a importância de discussões e abordagens que tenham como foco pessoas com deficiência, as TAs e as questões socioculturais que influenciam a forma como estas são percebidas e, consequentemente, tratadas. Afinal, é provável, que por relacionar a emoção tristeza ao objeto cadeira de rodas, as pessoas relacionem este sentimento e outros sentimentos negativos às pessoas que utilizam a cadeira de rodas. O que permite refletir que, embora haja atualmente um discurso igualitário e de inclusão, na realidade ainda tem-se a noção de TA como algo marginalizado.

Assim, mais do que questões relacionadas às Políticas Sociais, esta é também uma questão de interesse de áreas do conhecimento relacionadas ao desenvolvimento e aprimoramento de novas tecnologias, como Design. Afinal, estes produtos ainda possuem uma estética pouco trabalhada, possuindo características de equipamento médico hospitalar, quando na verdade são, para quem os utiliza, essenciais as atividades da vida diária e também para o contato social.

\section{Referências}

BISPO, Renato; BRANCO, Renato. Designing out stigma: the role of objects in the construction of disabled people's identity. In: Dare to Desire: 6th International Design and Emotion Conference. 2008.

BROOKS, N. A. Models for understanding rehabilitation and assistive technology. De- 
signing and using assistive technology. The human perspective, 1998, 3-11.

COSTA, Viviane de Souza Pinho, et al. Representaciones sociales de la silla de ruedas para la persona con lesión de la médula espinal. Revista Latinoamericana de Enfermagem, 2010, p. 755-762.

DESMET, Pieter; DIJKHUIS, Eva. A wheelchair can be fun: a case of emotion-driven design. In: Proceedings of the 2003 international conference on Designing pleasurable products and interfaces. ACM, 2003. p. 22-27.

FELLINGHAUER, Bernd AG et al. Construct validity, test-retest reliability, and internal consistency of the Photo Elicitation Semantic Differential scale (PESD) in disability studies. Journal of Developmental and Physical Disabilities, v. 23, n. 3, p. 257-265, 2011.

GETHING, Lindsay. Judgements by health professionals of personal characteristics of people with a visible physical disability. Social Science \& Medicine, v. 34, n. 7, p. 809815, 1992.

HERRERA-SARAY, Patricia et al. Problemas con el uso de sillas de ruedas y otras ayudas técnicas y barreras sociales a las que se enfrentan las personas que las utilizan. Estudio cualitativo desde la perspectiva de la ergonomía en personas discapacitadas por enfermedades reumáticas y otras condiciones. Reumatología Clínica, v. 9, n. 1, p. 24-30, 2013.

INSTITUTO DE MÉTRICA E AVALIAÇÃO EM SAÚDE. Carga da doença mundial. Universidade de Washington, p. 29, 2010.

ISO 9999:2002. Norma Internacional; classificação. Disponível em <http://www.inr. pt/content/1/59/ajudas-tecnicas/> Acesso em 05 ago. 2016.

LANUTTI, Jamille NL, et al. The significance of manual wheelchairs: a comparative study on male and female users. Procedia Manufacturing, 2015, vol. 3, p. 6079-6085.

LUBORSKY, Mark R. Sociocultural factors shaping technology usage: Fulfilling the promise. Technology and Disability, 1993, vol. 2, no 1, p. 71.

MEDOLA, Fausto Orsi. Desenvolvimento de um aro de propulsão manual ergonômico para cadeira de rodas. 2010. Tesis Doctoral. Universidade de São Paulo.

MERLEAU-PONTY, Maurice. Fenomenologia da percepção. (tradução Carlos Alberto Ribeiro de Moura). 2ª Ed. São Paulo: Martins Fontes, 1999, 662 p.

ORGANIZAÇÃO MUNDIAL DA SAÚDE. Relatório mundial sobre a deficiência. São Paulo: Lingüísticos, p. 5-29, 2011. 
PARETTE, Phil; SCHERER, Marcia. Assistive technology use and stigma. Education and Training in Developmental Disabilities, 2004, p. 217-226.

PHILLIPS, Betsy; ZHAO, Hongxin. Predictors of assistive technology abandonment. Assistive technology, 1993, 5.1: 36-45.

RIEMER-REISS, Marti L.; WACKER, Robbyn R. Factors associated with assistive technology discontinuance among individuals with disabilities. Journal of Rehabilitation, 2000, 66.3: 44.

SILVEIRA, Isabel Orestes. Design, Semiose e Ação comunicativa. Revista Tríades, v. 1, n. 2 (Julho), 2012, 19 p.

VAES, Kristof, et al. Stigma-Free Product Design: An Exploration in Dust Mask Design. In: DS 74: Proceedings of the 14th International Conference on Engineering \& Product Design Education (E\&PDE12) Design Education for Future Wellbeing, Antwerp, Belguim, 06-07.9. 2012. 2012.

VAES, Kristof, et al. Product Stigmaticity: Understanding, Measuring and Managing Product-Related Stigma. Kristof Vaes/Delft Academic Press, 2014.

VÁSQUEZ, Melissa Marín, et al. Avaliação da percepção estética, simbólica e de uso de cadeiras de rodas manuais. In: International Conference on Design,

Engineering, Management for innovation. 2015

VERZA, R., et al. An interdisciplinary approach to evaluating the need for assistive technology reduces equipment abandonment. Multiple sclerosis, 2006, 12.1: 88-93.

WANG, Ming-Hung et al. Coping style and personal responsibility as factors in the perception of individuals with physical disabilities by Chinese international students. Rehabilitation Psychology, v. 42, n. 4, p. 303, 1997.

WORLD HEALTH ORGANIZATION, et al. Pautas para el suministro de sillas de ruedas manuales en entornos de menores recursos. 2008. 